sufficient to absorb virtually all of the incident X-ray emission from the $\mathrm{AGN}^{22}$. This result, and the fact that the disk is viewed nearly edge-on, support the idea that the $\mathrm{HZ}$ traces ionized gas lying within the obscuring disk of NGC1068. One difficulty is that the covering fraction of the compact sources, $\sim 5-10 \%$, is much smaller than required generally to explain the fraction of directly viewed $\mathrm{AGNs}^{23}$ (the covering fraction is the portion of a sphere surrounding an AGN which is covered by obscuring clouds). However, the model can be reconciled if the geometric thickness of the obscuring medium increases with radius ${ }^{24}$, and the $\mathrm{HZ}$ traces only emission from disk material nearest the AGN. Alternatively, the obscuring disk might instead be thin but highly warped ${ }^{25}$, and again the HZ marks only the most central region of the disk.

Turning to the broad-band properties of the disk, the HZ plasma must also be a source of line and continuum emission up to soft X-ray energies. To determine whether the optical-X-ray spectrum of the HZ might be distinguished from neighbouring emission-line regions and the AGN proper, we modelled the HZ spectrum using the CLOUDY photoionization code ${ }^{26}$, programmed to emulate a cooling plasma with the properties of the $\mathrm{HZ}$, and normalized to the observed radio flux. We find that, in broad agreement with Pier and Voit ${ }^{7}$, the HZ contributes $\leqslant 10 \%$ to the observed optical-ultraviolet emission lines of NGC1068 and $\leqslant 1 \%$ to the optical-ultraviolet continuum. On the other hand, the HZ should contribute significantly to the soft X-ray spectrum of the nucleus were the AGN viewed along an unobscured sight-line. We estimate that free-free emission from the $\mathrm{HZ}$ may contribute $\geqslant 10 \%$ of the AGN continuum in soft X-rays (photon energies $\sim 1-2 \mathrm{keV})$. Moreover, the predicted soft X-ray line emission exceeds that observed towards $\mathrm{NGC1068} 8^{27}$ by a factor of $\geqslant 100$. This observed diminution is approximately that expected for obscured X-ray emission viewed only in reflection ${ }^{19}$. Therefore, this result can be reconciled if the $\mathrm{HZ}$ is also heavily obscured over optical-soft X-ray wavebands. The implication is that, if the AGN unifying schemes hold generally, the disks surrounding unobscured AGN should be luminous soft X-ray emission-line sources. To our knowledge, there has as yet been no analysis of the soft X-ray line emission from unobscured AGN (that is, Seyfert 1 AGN). The detection of soft X-ray lines characteristic of a $10^{6}-10^{7} \mathrm{~K}$ plasma in unobscured AGN would lend self-consistency to the obscuring disk model.

The present observation is, to our knowledge, the first direct image of a parsec-scale, ionized gas disk surrounding an AGN. Simple models for the radio emission further provide direct estimates of the physical properties of a parsec-scale disk, and the results are consistent with the predictions of AGN unifying schemes. However, we also find that photoionization heating is insufficient to support the high plasma temperatures in the disk, and so the challenge remains to model the energy budget in accord with the observed radio emission. Equally important is how the $\mathrm{HZ}$ might fit into the standard, infall model for $\mathrm{AGN}^{2}$. The $\mathrm{HZ}$ is orientated nearly at right angles to the radio jet. The observed orientation suggests that, within the HZ, internal, viscous dissipation drives the fuelling of the AGN rather than external torques. In this regard, the $\mathrm{HZ}$ may be considered to define the outer extent of the long-sought accretion disk powering the AGN.

Received 4 March; accepted 2 July 1997.

1. Rees, M. J. Black hole models for active galactic nuclei. Annu. Rev. Astron. Astrophys. 22, 471-506 (1984).

2. Gunn, J. E. in Active Galactic Nuclei (eds Hazard, C. \& Mitton, S.) 213-225 (Cambridge Univ. Press, 1979).

. Begelman, M. C., Blandford, R. D. \& Rees, M. J. Theory of extragalactic radio sources. Rev. Mod. Phys. 56, 255-351 (1984)

4. Lawrence, A. Classification of active galaxies and the prospect of a unified phenomenology. Publ. Astron. Soc. Pacif. 99, 309-334 (1987).

5. Antonucci, R. R. J. \& Miller, J. S. Spectropolarimetry and the nature of NGC 1068. Astrophys. J. 297, 621-532 (1985).

6. Krolik, J. H. \& Begelman, M. C. Molecular tori in Seyfert galaxies-Feeding the monster and hiding it. Astrophys. J. 329, 702-711 (1988).
7. Pier, E. A. \& Voit, G. M. Photoevaporation of dusty clouds near active galactic nuclei. Astrophys. J. 450, 628-637 (1995).

8. Gallimore, J. F., Baum, S. A. \& O'Dea, C. P. The subarcsecond radio structure in NGC1068. II. Implications for the central engine and unifying schemes. Astrophys. J. 464, 198-211 (1996).

9. Muxlow, T. W. B., Pedlar, A., Holloway, A. J., Gallimore, J. F. \& Antonucci, R. R. J. The compact radio nucleus of the Seyfert galaxy NGC1068. Mon. Not. R. Astron. Soc. 278, 854-860 (1996).

10. Gallimore, J. F., Baum, S. A., O'Dea, C. P., Brinks, E. \& Pedlar, A. $\mathrm{H}_{2} \mathrm{O}$ and $\mathrm{OH}$ masers as probes of the obscuring torus in NGC1068. Astrophys. J. 462, 740-745 (1996).

11. Antonucci, R. R. J., Hurt, T. \& Miller, J. S. HST ultraviolet spectropolarimetry of NGC1068. Astrophys. J. 430, 210-217 (1994).

12. Kellerman, K. I. \& Owen, F. N. in Galactic and Extragalactic Radio Astronomy (eds Verschuur, G. L. \& Kellermann, K. I.) 563-600 (Springer, New York, 1989).

13. Capetti, A., Macchetto, F., Axon, D. J., Sparks, W. B. \& Boksenberg, A. Hubble Space Telescope imaging polarimetry of the inner nuclear region of NGC 1068. Astrophys. J. 452, L87-L89 (1995).

14. Gallimore, J. F., Baum, S. A., O'Dea, C. P. \& Pedlar, A. The subarcsecond radio structure in NGC 1068 I. Observations and results. Astrophys. J. 458, 136-148 (1996).

15. Mezger, P. G., Henderson, A. P. Galactic H II regions: I. Observations of their continuum radiation at the frequency $5 \mathrm{GHz}$. Astrophys. J. 147, 471-489 (1967).

16. Neufeld, D. A., Maloney, P. R. \& Conger, S. Water maser emission from X-ray-heated circumnuclear gas in active galaxies. Astrophys. J. 436, L127-L130 (1994)

17. Krolik, J. H., McKee, C. F. \& Tarter, C. B. Two-phase models for quasar emission line regions. Astrophys. J. 249, 422-442 (1981).

18. Krolik, J. H. \& Begelman, M. C. An X-ray heated wind in NGC 1068. Astrophys. J. 308, L55-L58 (1986).

19. Pier, E. A., Antonucci, R., Hurt, T., Kriss, G. \& Krolik, J. The intrinsic nuclear spectrum of NGC 1068 Astrophys. J. 428, 124-129 (1994).

20. Reynolds, C. F. \& Fabian, A. C. Warm absorbers in active galactic nuclei. Mon. Not. R. Astron. Soc. 273, $1167-1176$ (1995).

21. Begelman, M. C. \& Fabian, A. C. Turbulent mixing layers in the interstellar and intracluster medium. Mon. Not. R. Astron. Soc. 244, 26P-29P (1991).

22. Mulchaey, J. S., Mushotzky, R. F. \& Weaver, K. A. Hard X-ray tests of the unified model for an ultraviolet-detected sample of Seyfert 2 galaxies. Astrophys. J. 390, L69-L72 (1992).

23. Lawrence, A. The relative frequency of broad-lined and narrow-lined active galactic nucleiImplications for unified schemes. Mon. Not. R. Astron. Soc. 252, 586-592 (1991).

24. Efstathiou, A., Hough, J. H. \& Young, S. A model for the infrared continuum spectrum of NGC 1068. Mon. Not. R. Astron. Soc. 277, 1134-1144 (1995).

25. Sanders, D. B., Phinney, E. S., Neugebauer, G., Soifer, B. T. \& Matthews, K. Continuum energy distribution of quasars - Shapes and origins. Astrophys. J. 357, 29-51 (1989).

26. Ferland, G. HAZY, a BriefIntroduction to Cloudy (Internal Rep., Dept of Physics and Astronomy, Univ. Kentucky, Lexington, 1993).

27. Ueno, S. et al. ASCA observations of NGC 1068. Publ. Astron. Soc. Jpn 46, L71-L75 (1994).

28. Cornwell, T. \& Braun, R. in Synthesis Imaging in Radio Astronomy (eds Perley, R. A., Schwab, F. R. \& Bridle, A. H.) 167-181 (ASP Conf. Ser., Astron. Soc. Pacif., San Francisco, 1994).

Acknowledgements. The VLBA and VLA are operated by the National Radio Astronomy Observatory which is operated by Associated Universities, Inc., under cooperative agreement with the NSF. J.F.G. received support from a Collaborative Visitor's Grant from the Space Telescope Science Institute.

Correspondence and requests for materials should be addressed to J.F.G. (e-mail: jfg@hethp. mpe-garching.mpg.de)

\section{Core formation on Mars and differentiated asteroids}

\section{Der-Chuen Lee and Alex N. Halliday}

Department of Geological Sciences, University of Michigan, Ann Arbor, Michigan 48109-1063, USA

Meteorite chronometry based on the ${ }^{182} \mathrm{Hf}-{ }^{182} \mathrm{~W}$ system can provide powerful constraints on the timing of planetary accretion and differentiation ${ }^{1-4}$, although the full potential of this method has yet to be realized. For example, no measurements have been made on the silicate-rich portions of planets and planetesimals other than the Earth and Moon. Here we report tungsten isotope compositions for two eucrites, thought to be derived from asteroid 4 Vesta, and from eight other basaltic achondritic meteorites that are widely considered to be from Mars. The eucrites, which are among the oldest differentiated meteorites, yield exceedingly radiogenic tungsten, indicating rapid accretion, differentiation and core formation on Vesta within the first 5$15 \mathrm{Myr}$ of Solar System history, whereas the range of radiogenic tungsten measurements on the martian meteorites points towards tungsten depletion via melting and core formation within the first $30 \mathrm{Myr}$ of the Solar System. The survival of tungsten isotope heterogeneity in the martian upper mantle implies that no giant impacts or large-scale convective mixing took place since this time. These results contrast with those obtained for the EarthMoon system ${ }^{2,3}$ for which accretion and core formation related to 
giant impacts appears to have continued for at least an additional 20 Myr.

Hafnium (Hf) and tungsten (W) are refractory elements whose relative proportions are strongly fractionated between silicate and metal. ${ }^{182} \mathrm{Hf}$ decays with a half-life of $9 \mathrm{Myr}$ to ${ }^{182} \mathrm{~W}$ such that the $\mathrm{W}$ isotope composition will be a function of the timing of $\mathrm{Hf} / \mathrm{W}$ fractionation, and hence that of silicate-metal segregation ${ }^{1-4}$. Despite a high Hf/W ratio, the silicate Earth has a W isotope composition identical to that of carbonaceous chondrites ${ }^{4}$, implying that the Earth's present core did not form until $>50 \mathrm{Myr}$ after the formation of the Solar System ${ }^{2,3}$ unless accretion was slower than generally modelled ${ }^{3}$. Iron meteorites and metal separates of ordinary chondrites contain $\mathrm{W}$ which is less radiogenic than chondritic, consistent with early segregation ${ }^{4}$. Tungsten which is more radiogenic than chondritic should be present in the silicate portions of some planets and planetesimals. Here we report results obtained on basaltic achondrites, thought to be derived from asteroid 4 Vesta and Mars. The siderophile element abundance patterns for both bodies are consistent with homogeneous accretion and core-mantle equilibration, possibly in a magma ocean ${ }^{5}$, in contrast to the non-equilibrium 'late veneer' model widely invoked to explain the excess siderophile concentrations in chondritic relative proportions that characterize the silicate Earth $^{6}$.

Eucrites represent basaltic magmas erupted at, or near, the surface of the eucrite parent body (EPB), thought to be asteroid 4 Vesta (ref. 7). ${ }^{60} \mathrm{Fe}-{ }^{60} \mathrm{Ni},{ }^{53} \mathrm{Mn}-{ }^{53} \mathrm{Cr}$ and $\mathrm{Pu}-\mathrm{Xe}$ data indicate that Vesta formed within the first $\sim 20 \mathrm{Myr}$ of the Solar System ${ }^{8-11}$. The W isotope composition of two eucrites, ALHA78132 and Juvinas, are extremely radiogenic, corresponding to high, but different, Hf/W ratios (Table 1) and reflecting in situ ${ }^{182} \mathrm{Hf}$ decay within the EPB in overlapping stages: (1) accretion of the EPB with chondritic Hf/W; (2) metal segregation producing a silicate mantle with high $\mathrm{Hf} / \mathrm{W}$;

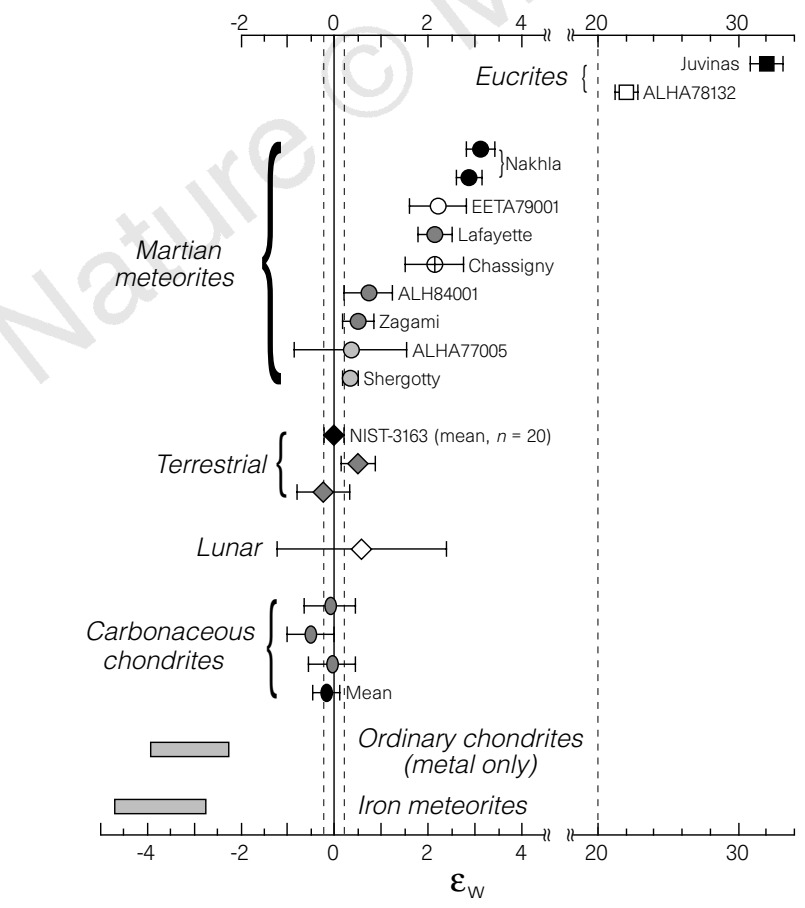

Figure $1 \epsilon_{\mathrm{w}}$ values of the two eucrites and eight martian meteorites analysed in this study $\left(\right.$ Table $1 ; \epsilon_{\mathrm{W}}=\left[\left(\left({ }^{182} \mathrm{~W} /{ }^{184} \mathrm{~W}\right)_{\text {meas }} /\left({ }^{182} \mathrm{~W} /{ }^{184} \mathrm{~W}\right)_{\text {std }}\right)-1\right] \times 10^{4}$, relative to the NIST-3163 standard). Also plotted are previously reported data ${ }^{1-4}$ for comparison. Duplicates are shown with the same symbols. The ranges of $\epsilon_{\mathrm{w}}$ values for the metal separates of ordinary chondrites and iron meteorites do not include the analytical uncertainties of individual samples.
(3) melting to form a magma and rock with $\mathrm{Hf} / \mathrm{W}$ slightly dissimilar from its source ${ }^{12}$. A Hf-W model age $\left(T_{\mathrm{CHUR}}\right)$ can be calculated as follows:

$$
T_{\text {CHUR }}=\frac{1}{\lambda} \ln \left\{\left(\frac{{ }^{82} \mathrm{Hf}}{{ }^{180} \mathrm{Hf}}\right)_{\mathrm{BSSI}} \times\left[\frac{\left(\frac{180 \mathrm{Hf}}{184 \mathrm{~W}}\right)_{\text {sample }}-\left(\frac{{ }^{80} \mathrm{Hf}}{{ }^{184} \mathrm{~W}}\right)_{\text {chond }}}{\left(\frac{82}{184}\right)_{\text {sample }}-\left(\frac{{ }^{82} \mathrm{~W}}{{ }^{184} \mathrm{~W}}\right)_{\text {chond }}}\right]\right\}
$$

where $\lambda$ is the decay constant of ${ }^{182} \mathrm{Hf}\left(\sim 0.077 \mathrm{Myr}^{-1}\right)$; $\left({ }^{182} \mathrm{Hf} /{ }^{180} \mathrm{Hf}\right)_{\mathrm{BSSI}}$ is the initial isotopic composition of the Solar system, $(2.4 \pm 0.6) \times 10^{-4}$ (refs 2,4$)$; and chond indicates chondritic. This equation provides the age of metal segregation if $\mathrm{Hf} / \mathrm{W}$ fractionation takes place in a single event. The two eucrites studied here yield similar $\mathrm{Hf}-\mathrm{W} T_{\mathrm{CHUR}}$ model ages in the range $5-15 \mathrm{Myr}$ (Table 1), consistent with early metal segregation. More complex models such as concomitant core formation and accretion ${ }^{3}$ are also consistent with the $\mathrm{W}$ data, provided that the mean time for accretion is of the order of 3 Myr. No matter how complicated the model, the $\mathrm{W}$ isotope data appear inconsistent with the completion of metal segregation $<4$ Myr or $>16$ Myr after the start of the Solar System. This is in excellent agreement with the timescales inferred from ${ }^{60} \mathrm{Fe}-{ }^{60} \mathrm{Ni}$ data (ref. 8), but is more difficult to reconcile with recent interpretations of ${ }^{53} \mathrm{Mn}-{ }^{53} \mathrm{Cr}$ data (ref. 10). The absolute age of a eucrite can be determined by comparing its initial ${ }^{53} \mathrm{Mn}$ abundance with that of angrites, the most precisely dated of all meteorites ${ }^{13}$. On this basis the age of Juvinas has been estimated to be 4,562 \pm 1 Myr, only 0.5-6.5 Myr later than the formation of the first condensates of the solar nebula ${ }^{14}$, just overlapping the timescale inferred from Hf-W. No independent age estimates are available for ALHA78132. However, the $\mathrm{Cr}$ isotope compositions of eucrites correlate with their respective $\mathrm{Mn} / \mathrm{Cr}$ ratios in a manner interpreted to reflect primarily source heterogeneity inherited from planetaryscale partial melting at 4,565 $\pm 1 \mathrm{Myr}$ (ref. 10), slightly earlier than allowed by the Hf-W model ages and inferred from ${ }^{60} \mathrm{Fe}-{ }^{60} \mathrm{Ni}$. Determination of detailed internal Hf-W isochrons should allow a direct comparison with $\mathrm{Mn}-\mathrm{Cr}$ data and the resolution of this disparity.

SNC meteorites (shergottites, nakhlites and Chassigny) are young $(<2$ Gyr $)$ achondrites which share chemical and oxygen isotope compositions consistent with a single large parent body, most likely to be $\mathrm{Mars}^{6,15}$. An older meteorite, ALH84001, is different from the SNC meteorites, but seems to be from the same planet. ALH84001 and three shergottites (ALHA77005, Shergotty and Zagami) yield

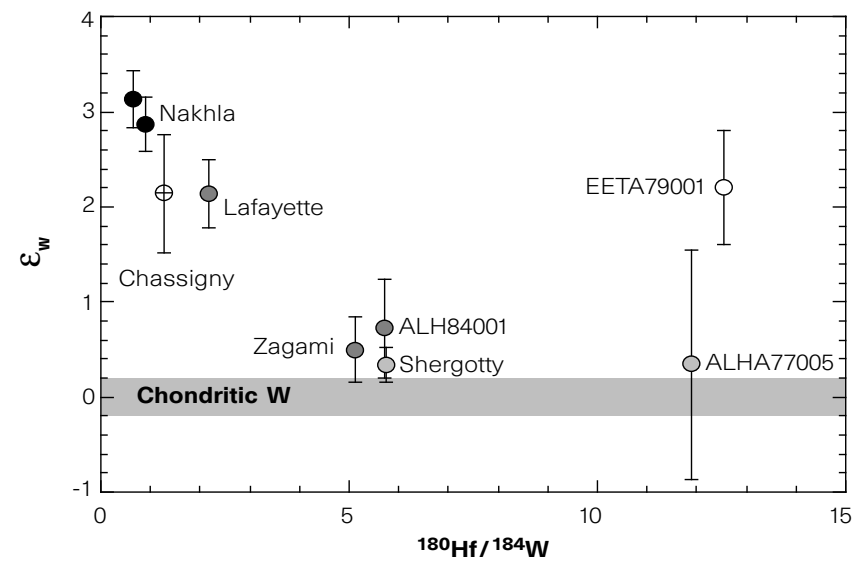

Figure $2{ }^{180} \mathrm{Hf} /{ }^{184} \mathrm{~W}$ versus $\epsilon_{\mathrm{W}}$ for eight martian meteorites measured in this study (Table 1), including a duplicate measurement of Nakhla. The horizontal shaded bar indicates the uncertainty over the $\mathrm{W}$ isotopic composition of carbonaceous chondrites. 


\begin{tabular}{|c|c|c|c|c|c|c|}
\hline Sample & $\begin{array}{c}\mathrm{Hf} \\
\text { (p.p.m.) }\end{array}$ & $\begin{array}{c}\text { W } \\
\text { (p.p.m.) }\end{array}$ & $\begin{array}{c}{ }^{180} \mathrm{Hf} /{ }^{184} \mathrm{~W} \\
\text { (atomic) }\end{array}$ & $\begin{array}{l}{ }^{182} \mathrm{~W} /{ }^{184} \mathrm{~W} \\
\pm 2 \sigma \text { error }\end{array}$ & $\begin{array}{c}\epsilon_{W} \\
\pm 2 \sigma \text { error }\end{array}$ & $\begin{array}{l}T_{\text {CHUR }} \\
\text { (Myr) }\end{array}$ \\
\hline \multicolumn{7}{|l|}{ Eucrites } \\
\hline ALHA78132 & 1.014 & 0.07200 & 16.61 & $0.866901 \pm 66$ & $+22.0 \pm 0.8$ & $8_{-4}^{+3}$ \\
\hline Juvinas (USNM2844) & 1.321 & 0.05606 & 27.81 & $0.86790 \pm 17$ & $+33.5 \pm 2.0$ & $10_{-4}^{+3}$ \\
\hline \multicolumn{7}{|l|}{ SNC meteorites } \\
\hline ALH84001 & 0.2152 & 0.04449 & 5.707 & $0.865062 \pm 45$ & $+0.72 \pm 0.52$ & $26_{-23}^{+8}$ \\
\hline ALHA77005 & 0.5586 & 0.05543 & 11.89 & $0.86503 \pm 10$ & $+0.34 \pm 1.2$ & $33_{-33}^{+16}$ \\
\hline Shergotty (ME1372) & 1.856 & 0.3804 & 5.756 & $0.865029 \pm 16$ & $+0.34 \pm 0.28$ & $33_{-24}^{+8}$ \\
\hline Zagami & 1.946 & 0.4449 & 5.110 & $0.865043 \pm 29$ & $+0.50 \pm 0.34$ & $29_{-23}^{+8}$ \\
\hline EETA79001 & 0.8198 & 0.07709 & 12.55 & $0.865191 \pm 52$ & $+2.21 \pm 0.60$ & $13_{-13}^{+16}$ \\
\hline Lafayette (ME2116) & 0.2801 & 0.1512 & 2.185 & $0.865185 \pm 31$ & $+2.14 \pm 0.36$ & $13_{-10}^{+6}$ \\
\hline Nakhla-1 (USNM5891) & 0.2040 & 0.2641 & 0.9112 & $0.865248 \pm 24$ & $+2.87 \pm 0.28$ & $10_{-9}^{+5}$ \\
\hline Nakhla-2 (USNM5891) & 0.1641 & 0.2976 & 0.6506 & $0.865271 \pm 26$ & $+3.13 \pm 0.30$ & $9_{-9}^{+5}$ \\
\hline Chassigny & 0.04435 & 0.04106 & 1.275 & $0.865185 \pm 54$ & $+2.14 \pm 0.62$ & $13_{-11}^{+6}$ \\
\hline
\end{tabular}

The differences in $\mathrm{Hf}$ and $\mathrm{W}$ concentrations in the two separately crushed aliquots of Nakhla are probably due to sample heterogeneity. The $\epsilon_{\mathrm{w}}$ of each $\mathrm{W}$ isotope measurement is expressed as the deviations in parts per $10^{4}$ relative to the NIST-3163 W standard, which gives a ${ }^{182} \mathrm{~W}^{184} \mathrm{~W}=0.865000 \pm 18(n=20)$. $T_{\text {CHUR }}$ is defined in equation (1).

W which is close to chondritic $\left(\epsilon_{\mathrm{w}}=0\right.$; see Fig. 1 for definition of $\left.\epsilon_{\mathrm{w}}\right)$, whereas two nakhlites (Nakhla and Lafayette), one shergottite (EETA79001) and Chassigny all display excess ${ }^{182} \mathrm{~W}\left(\epsilon_{\mathrm{w}}=+2\right.$ to +3 ; Table 1; Fig. 1).

ALH84001 is estimated to be $>4$ Gyr in age (ref. 16), but it must be younger than $4.53 \mathrm{Gyr}$ given its chondritic $\mathrm{W}$ isotope composition. All other martian meteorites are relatively young igneous rocks ${ }^{17-20}$ and the measured $\mathrm{Hf} / \mathrm{W}$ ratio, the complex product of a long history of melting, is clearly unrelated to the $\mathrm{W}$ isotope compositions which only relate to the earliest development of Mars (Fig. 2). It is, therefore, necessary to use the ${ }^{180} \mathrm{Hf} /{ }^{184} \mathrm{~W}$ of $4 \pm 1$ for the primitive martian mantle (PMM) (refs 5, 21) in order to extract age information. This $\mathrm{Hf} / \mathrm{W}$ ratio is much smaller than that estimated for the $\mathrm{EPB}^{5}$, so early core formation has not produced $\mathrm{W}$ which is as radiogenic as is found in the eucrites (Fig. 1). The samples with $\mathrm{W}$ close to chondritic yield imprecise model ages when calculated in this fashion (Table 1). However, those with radiogenic $\mathrm{W}$ yield $\mathrm{Hf}-\mathrm{W} T_{\mathrm{CHUR}}$ model ages indicative of early differentiation. The data for Nakhla indicate differentiation within the first $15 \mathrm{Myr}$ of the Solar System. Modelling these data in terms of concomitant accretion and core formation $^{3}$ yields a mean accretionary time of $5 \mathrm{Myr}$ for Mars. The $\mathrm{W}$ isotope compositions could have been generated in an environment with higher $\mathrm{Hf} / \mathrm{W}$ than the PMM, but the constraints

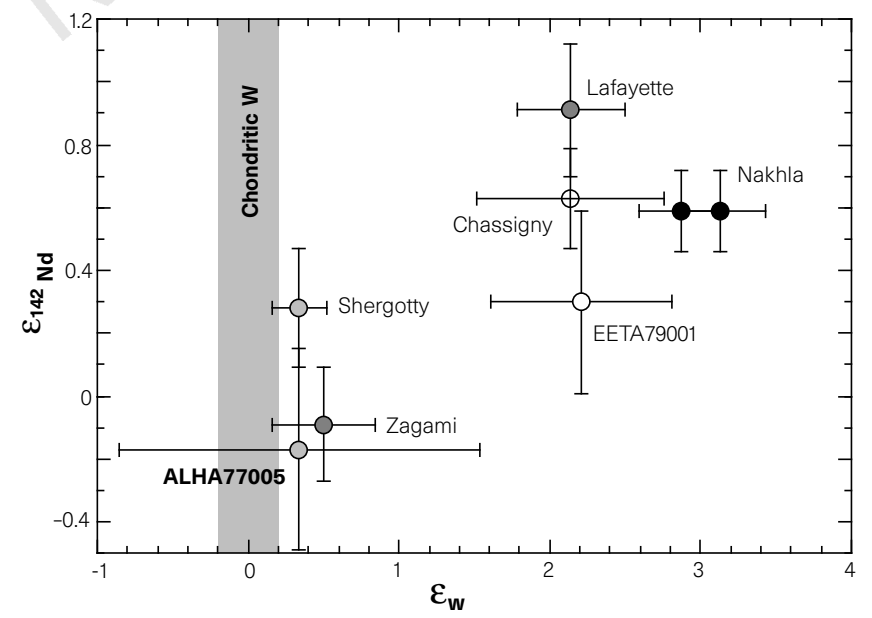

Figure $3 \epsilon_{\mathrm{W}}$ versus $\epsilon_{142} \mathrm{Nd}$ for the martian meteorites analysed in this study. The $\epsilon_{142} \mathrm{Nd}$ data are taken from Harper et al. (ref. 22). $\epsilon_{142 \mathrm{Nd}}$ is the deviation in parts per $10^{4}$ of the ${ }^{142} \mathrm{Nd} /{ }^{144} \mathrm{Nd}$ ratio from the terrestrial values. The vertical shaded bar indicates the uncertainty for the $\mathrm{W}$ isotopic composition of carbonaceous chondrites. remain tight. Even if the Hf/W ratio were as high as EETA79001, three times the PMM, the data would still imply differentiation within the first $30 \mathrm{Myr}$.

These constraints from $\mathrm{W}$ isotopes are consistent with other isotope data for martian meteorites which also suggest early planetary-scale differentiation ${ }^{15-20,22}$. Most importantly, the W data are in excellent agreement with data for ${ }^{146} \mathrm{Sm}-{ }^{142} \mathrm{Nd}$ (halflife, $103 \mathrm{Myr}$ ) from the same martian meteorites ${ }^{22}$ (Fig. 3), also indicative of rapid accretion and differentiation. Those with excess ${ }^{142} \mathrm{Nd}$, reflecting early depletion in light rare earths and other incompatible elements, are characterized by radiogenic W (Fig. 3 ). In order to generate a positive correlation between the $\mathrm{W}$ and $\mathrm{Nd}$ isotope data, the two parent/daughter ratios must have fractionated together at an early stage. If the core of Mars formed before the silicate melt depletion responsible for the high $\mathrm{Sm} / \mathrm{Nd}$, the enhanced Hf/W ratio of the silicate Mars would have driven the $\mathrm{W}$ isotope data to greater than chondritic values while the martian mantle maintained a chondritic $\mathrm{Sm} / \mathrm{Nd}$ ratio and $\mathrm{Nd}$ isotope composition. This is not observed, as martian samples with chondritic Nd also have chondritic W isotope compositions (Fig. 3). Conversely, if core formation on Mars were later than silicate melt depletion, the presence of a metal phase during silicate melting would have buffered the concentrations of $\mathrm{W}$ and resulted in an inverse, or lack of, correlation between $\mathrm{Sm} / \mathrm{Nd}$ and $\mathrm{Hf} / \mathrm{W}$ in the melts. The positive correlation between $\mathrm{Nd}$ and $\mathrm{W}$ isotope data is also inconsistent with this model. The simplest explanation is that silicate melting and metal segregation (core formation) were coeval and co-genetic. Such a close relationship in a rapidly accreting planet is scarcely surprising as accretional energy and the decay of short-lived nuclides would have quickly heated the planet ${ }^{5}$, resulting in the formation of a shallow magma ocean from which metal segregated $^{5,23}$, as also indicated by metal-silicate equilibria for moderate siderophile elements ${ }^{5}$.

Although internal heating through the decay of $\mathrm{U}$, Th and $\mathrm{K}$ will sustain melting, the occurrence of long-lived, large-scale vigorous mantle convection, as in the Earth, would effectively homogenize $\mathrm{W}$ isotope differences in the source regions of martian meteorites. Therefore, plate tectonics on Mars ${ }^{24}$ would seem to be inconsistent with the $\mathrm{W}$ isotope data. This is consistent with the clear dichotomy between the ages of crust in the northern and southern hemispheres ${ }^{25}$, the fact that ${ }^{40} \mathrm{Ar}$ is undegassed ${ }^{20,25}$, a dynamically layered mantle $e^{26}$ with perovskite at the base $\mathrm{e}^{27}$, and melting and tectonics driven by a single plume ${ }^{28}$.

The ${ }^{142} \mathrm{Nd} /{ }^{144} \mathrm{Nd}$ and ${ }^{182} \mathrm{~W} /{ }^{184} \mathrm{~W}$ ratios thus far measured have been only chondritic or radiogenic, implying melt and metal depletion, albeit with subsequent partial re-enrichment. Although meteorite sampling may not be representative, the outer portions of Mars may be enriched in the cumulate residues of a magma ocean. 
This would involve very dense Fe-rich melts ${ }^{15}$, particularly before the completion of core formation, and some of these melts may have partially sunk with core-forming metal towards the martian interior. Cumulate and residue flotation depends on the density crossover between solid and liquid phases ${ }^{27}$, which is poorly constrained for Mars. However, if the outer portions of the planet were rich in depleted residual solids during the magma ocean stage, the $\mathrm{W}$ and $\mathrm{Nd}$ isotope compositions would be expected to be radiogenic and to survive homogenization.

The sizes of inner Solar System planetary bodies seem to increase with accretion and differentiation intervals, from the most primitive and undifferentiated materials such as equilibrated chondrites $(\sim 6 \mathrm{Myr})$ to the eucrite parent body $(\sim 10 \mathrm{Myr})$ to Mars $(10-$ $30 \mathrm{Myr}$ ) and to the Earth-Moon system (>50 Myr) (refs 2, 3). The data are consistent with early termination of accretion of asteroids and Mars, and longer-lived accretion of larger bodies resulting from late planet-scale impacts. The trend corresponds to a transition in the dominant mechanisms of heating from decay of short-lived nuclides to the release of accretional energy. Whereas a late giant impact on Earth may have been sufficiently energetic to effectively homogenize the $\mathrm{W}$ isotope composition and destroy any protocore $^{2,3}$, the $\mathrm{W}$ and $\mathrm{Nd}$ isotope data are inconsistent with an impact sufficiently energetic to melt and homogenize Mars later than 4.53 Gyr (ref. 22).

Methods. All samples were powdered in an aluminum oxide mortar after surface saw marks and fusion crusts had been removed through mild acid leaching $(1 \mathrm{M} \mathrm{HCl})$ and hand-picking. The samples were digested sequentially with concentrated $\mathrm{HF}, 8 \mathrm{M} \mathrm{HNO}_{3}$ and $6 \mathrm{M} \mathrm{HCl}$. Roughly $10 \%$ of the solution was separated and spiked with ${ }^{178} \mathrm{Hf}$ and ${ }^{186} \mathrm{~W}$, whereas the remaining solution was dried and redissolved in $\sim 8 \mathrm{ml}$ of $4 \mathrm{M}$ HF. The method of chemical separation of $\mathrm{W}$ was adapted from the first column of the Hf chemistry developed by Salters and Hart ${ }^{29}$ but on a reduced scale, with $3.5 \mathrm{ml}$ of Bio-Rad AG1 $\times 8$ (200-400 mesh) anion resin. The Hf and W were eluted and collected sequentially using a mixed solution of $6 \mathrm{M} \mathrm{HCl}+1 \mathrm{M}$ HF. The same chemical procedure was used for the spiked solutions, but the column volume was further reduced $(\sim 1 \mathrm{ml})$, and $\mathrm{Hf}$ and $\mathrm{W}$ were collected together. The total $\mathrm{W}$ procedural blank was $\sim 0.4 \mathrm{ng}$. Tungsten isotopic measurement by multiple collector inductively coupled-plasma mass spectrometry (MC-ICPMS) has been previously described by Lee and Halliday ${ }^{30}$. The NIST-3163 W standard was run between every sample to monitor the performance of MC-ICPMS and to check for memory effects, which were negligible. The $\mathrm{W}$ isotopic measurements were normalized to ${ }^{186} \mathrm{~W} /{ }^{184} \mathrm{~W}=0.927633$ (ref. 30). The quoted $2 \sigma$ standard errors all refer to the least significant figures. The concentrations of $\mathrm{Hf}$ and $\mathrm{W}$ were determined by isotope dilution, and the uncertainty is typically $0.2 \%$ or better. All the $\mathrm{W}$ data are blank-corrected, which is negligible for all analyses except for Juvinas which has been corrected by $1.5 \epsilon$ unit.

Received 3 April; accepted 15 July 1997.

1. Harper. C. L. \& Jacobsen, S. B. Evidence for ${ }^{182} \mathrm{Hf}$ in the early Solar System and constraints on the timescale of terrestrial accretion and core formation. Geochim. Cosmochim. Acta 60, 1131-1153 (1996).

2. Lee, D.-C. \& Halliday, A. N. Hafnium-tungsten chronometry and the timing of terrestrial core formation. Nature 378, 771-774 (1995)

. Halliday, A. N., Rehkämper, M., Lee, D.-C. \& Yi, W. Early evolution of the Earth and Moon: new constraints from Hf-W isotope geochemistry. Earth Planet. Sci. Lett. 142, 75-89 (1996).

4. Lee, D.-C. \& Halliday, A. N. Hf-W isotopic evidence for rapid accretion and differentiation in the early solar system. Science 274, 1876-1879 (1996).

. Righter, K. \& Drake, M. J. Core formation in Earth's Moon, Mars and Vesta. Icarus 124, 513-529 (1996)

6. Wänke, H. \& Dreibus, G. Chemical composition and accretion history of terrestrial planets. Phil. Trans. R. Soc. Lond. A 325, 545-557 (1988).

. Binzel, R. P. \& Xu, S. Chips off of asteroid 4 Vesta: evidence for the parent body of basaltic achondrite meteorites. Science 260, 186-191 (1993).

8. Shukolyukov, A. \& Lugmair, G. W. ${ }^{60} \mathrm{Fe}$ in eucrites. Earth Planet. Sci. Lett. 119, 159-166 (1993).

9. Lugmair, G. W. \& MacIsaac, Ch. Radial heterogeneity of ${ }^{53} \mathrm{Mn}$ in the early solar system? Lunar Planet. Sci. XXVI, 879-880 (1995).

10. Lugmair, G. W. \& Shukolyukov, A. ${ }^{53} \mathrm{Mn}-{ }^{53} \mathrm{Cr}$ isotope systematics of the HED parent body. Lunar Planet. Sci. XXVIII, 651-852 (1997).

11. Shukolyukov, A. \& Begemann, F. Pu-Xe dating of eucrites. Geochim. Cosmochim. Acta 60, 2453-2471 (1996).

12. Newsom, H. E., White, W. M., Jochum, K. P. \& Hofmann, A. W. Siderophile and chalcophile elemen abundances in oceanic basalts, $\mathrm{Pb}$ isotope evolution and growth of the earth's core. Earth Planet. Sci. Lett. 88, 299-313 (1986).
13. Lugmair, G. W. \& Galer, S. J. G. Age and isotopic relationships among the angrites Lewis Cliff 86010 and Angra dos Reis. Geochim. Cosmochim. Acta 56, 1673-1694 (1992).

14. Göpel, C., Manhès, G. \& Allègre, C. J. Constraints on the time of accretion and thermal evolution of chondrite parent bodies by precise U-Pb dating of phosphates. Meteorites 26, 73 (1991).

15. McSween, H. Y. What we have learned about Mars from SNC meteorites. Meteoritics 29, 757-779 (1994)

16. Jagoutz, E., Sorowka, A., Vogel, J. D. \& Wänke, H. ALH84001: alien or progenitor of the SNC family? Meteoritics 9, 478-479 (1994).

17. Nakamura, N., Unruh, D. M. \& Tatsumoto, M. Origin and evolution of the Nakhla meteorite inferred from the $\mathrm{Sm}-\mathrm{Nd}$ and $\mathrm{U}-\mathrm{Pb}$ systematics and $\mathrm{REE}, \mathrm{Ba}, \mathrm{Sr}, \mathrm{Rb}$ and $\mathrm{K}$ abundances. Geochim. Cosmochim. Acta 46, 1555-1573 (1982).

18. Shih, C.-Y. et al. Chronology and petrogenesis of young achondrites, Shergotty, Zagami, and ALHA77005: late magmatism on a geologically active planet. Geochim. Cosmochim. Acta 46, $2323-$ 2344 (1982).

19. Chen, J. H. \& Wasserburg, G. J. Formation ages and evolution of Shergotty and its parent planet from U-Th-Pb systematics. Geochim. Cosmochim. Acta 50, 955-968 (1986).

20. Jagoutz, E. \& Wänke, H. Sr and Nd isotopic systematics of Shergotty meteorites. Geochim. Cosmochim. Acta 50, 939-953 (1986)

21. Treiman, A. H. et al. Core formation in the Earth and shergottite parent body (SPB): chemical evidence from basalts. Geochim. Cosmochim. Acta 50, 1071-1091 (1986).

22. Harper, C. L. et al. Rapid accretion and early differentiation of Mars indicated by ${ }^{142} \mathrm{Nd} /{ }^{144} \mathrm{Nd}$ in SNC meteorites. Science 267, 213-217 (1995).

23. Stevenson, D. J. in Origin of the Earth (eds Newsom, H. E. \& Jones, J. H.) 231-249 (Oxford Univ. Press, London, 1990).

24. Sleep, N. H. Martian plate tectonics. J. Geophys. Res. 99, 5639-5655 (1994).

25. Breuer, D., Spohn, T. \& Wüllner, U. Mantle differentiation and the crustal dichotomy of Mars. Planet. Space Sci. 41, 269-283 (1993).

26. Breuer, D., Yuen, D. A. \& Spohn, T. Phase transitions in the Martian mantle: implications for partially layered convection. Earth Planet. Sci. Lett. 148, 457-469 (1997).

27. Bertka, C. M. \& Fei, Y. Mineralogy of the Martian interior up to core-mantle boundary pressures. J. Geophys. Res. 102, 5251-5264 (1997).

28. Harder, H. \& Christensen, U. R. A one-plume model of martian mantle convection. Nature 380, $507-$ 509 (1996).

29. Salters, V. J. M. \& Hart, S. R. The mantle sources of ocean ridges, islands and arcs: the Hf-isotope connection. Earth Planet. Sci. Lett. 104, 364-380 (1994).

30. Lee, D.-C. \& Halliday, A. N. Precise determinations of the isotopic compositions and atomic weights of molybednum, tellurium, tin and tungsten using ICP magnetic sector multiple collector mass spectrometry. Int. J. Mass Spec. Ion Proc. 146/147, 35-46 (1995).

Acknowledgements. We thank M. Lindstrom, L. Nyquist, G. MacPherson, C. Perron and M. Wadhwa for access to their meteorite collections at NASA, Smithsonian Institution of Washington, Museum National d'Histoire Naturelle at Paris, and Field Museum in Chicago. We also thank J. Christensen, E. Essene, H. Pollack, M. Rehkämper, P. van Keken and Y. Zhang for their comments, M. Johnson and C. Hall for their assistance, and K. Righter and M. Drake for access to unpublished papers. This work was supported by NSF, DOE, NASA and the University of Michigan.

\section{Effect of microgravity on the} crystallization of a selfassembling layered material

\section{Homayoun Ahari, Robert L. Bedard $\dagger$, Carol L. Bowes*, Neil Coombs $\ddagger$ Ömer Dag*, Tong Jiang*, Geoffrey A. Ozin*, Srebri Petrov*, Igor Sokolov*, Atul Verma ${ }^{\star}$, Gregory Vovk ${ }^{\star}$ \& David Young*}

* Materials Chemistry Research Group, Lash Miller Chemical Laboratories, University of Toronto, Toronto, Ontario M5S 3H6, Canada

$\dagger$ UOP, Research Division, 25 E. Algonquin Road, Des Plaines, Illinois 60017, USA $\ddagger$ Imagetek Analytical Imaging, 32 Manning Avenue, Toronto, Ontario M6J 2K4, Canada

In microgravity, crystals of semiconductors and proteins can be grown with improved crystallinity, offering the prospect of improved structural analyses (for proteins) and better electronic properties (for semiconductors) ${ }^{1-3}$. Here we study the effect of a microgravity environment on the crystallization of a class of materials-layered microporous tin(IV) sulphides ${ }^{4-11}$ - whose crystal structure is determined by weak interlayer interactions (electrostatic, hydrogen-bonding and van der Waals) as well as strong intralayer covalent bonds. We find that the crystals grown in microgravity (on board the Space Shuttle Endeavour) show improved crystal habits, smoother faces, greater crystallinity, better optical quality and larger void volumes than the materials grown on Earth. These differences are due at least in part to the 